\title{
HCPortal Overview
}

\author{
Eugen Antal \\ Slovak University of \\ Technology in Bratislava \\ Slovakia \\ eugen.antal@stuba.sk
}

\author{
Pavol Zajac \\ Slovak University of \\ Technology in Bratislava \\ Slovakia \\ pavol.zajac@stuba.sk
}

\begin{abstract}
HCPortal is a portal consisting of several web pages and tools focusing on historical cryptology. The heart of the project is a comprehensive database of cryptograms accessible for everybody. The front-end of this portal was designed to provide a responsive and modern UI/UX. We used technologies built for the modern web. The major part of the portal's back-end is also available as a public API.
\end{abstract}

\section{Introduction}

The Portal of Historical Ciphers (HCPortal) is a gateway to the world of historical ciphers. You can find a comprehensive database of cryptograms, framework for document analysis, glossary and many more.

This project was created by researchers and students from the Slovak University of Technology in Bratislava in cooperation with other crypto history enthusiasts.

\section{The Portal}

The HCPortal consists of several parts. The portal's home page serves as an entry point, connecting these parts together. While the portal has started only recently, we have already prepared:

- Home page - entry point of the portal with navigation and information centre.

- Database of cryptograms - database with a public API, also contains visualization (frontend) and advanced search.

- ManuLab and ManuLab online - software product for statistical analysis, with a public API and example web page.
- Tools and web pages - links to external projects.

- Glossary - glossary for historical cryptology.

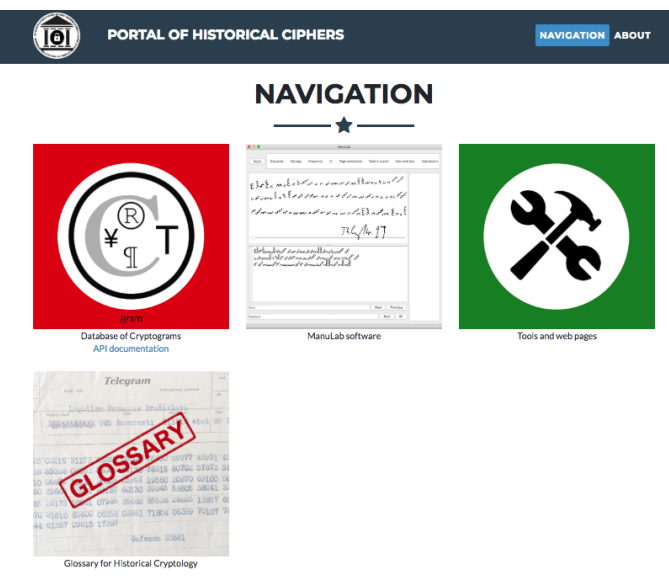

Figure 1: The main navigation screen.

The portal's entry point is available at: https://hcportal.eu/.

\section{Database of Cryptograms}

We are carefully collecting ${ }^{1}$ the most important information about known cryptograms, which are stored in a relational database. Cryptogram descriptions are also available through a web-service (public API). The front-end (web) contains cipher detail visualization and full-text search. We have also implemented an advanced search, where it is possible to find cryptograms based on location, language, sender and other parameters.

\footnotetext{
${ }^{1}$ The cryptograms are collected (and are planned) mainly from (Klausis Krypto Kolumne, 2019), (Crypto Cellar Research, 2019), (Breaking German Navy Ciphers, 2019), The Slovak National Archive and The Military History Archive of Slovakia, all with permissions.
} 
The API documentation is available at: https://www.cryptograms.hcportal.eu/ api/apidoc/index.html and accessible from: https://cryptograms.hcportal.eu.

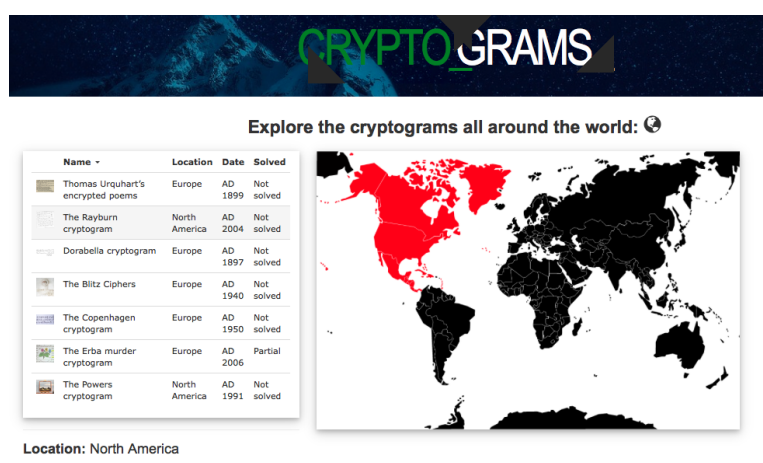

Figure 2: Cryptograms - home screen.

\section{HCPortal Home Cryptograms- Search-}



Figure 3: Cryptograms - main menu.

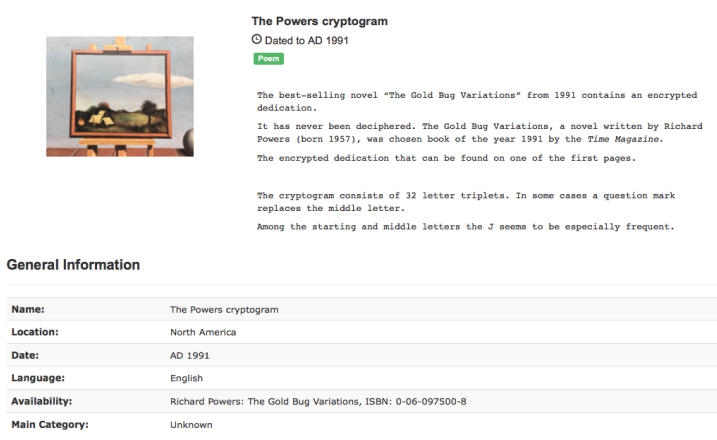

Figure 4: Cryptograms - cryptogram detail.

\section{ManuLab}

ManuLab is a software product for statistical analysis of encrypted historical manuscripts. The document analysis is performed via a chain of filters (main building elements). A filter represents

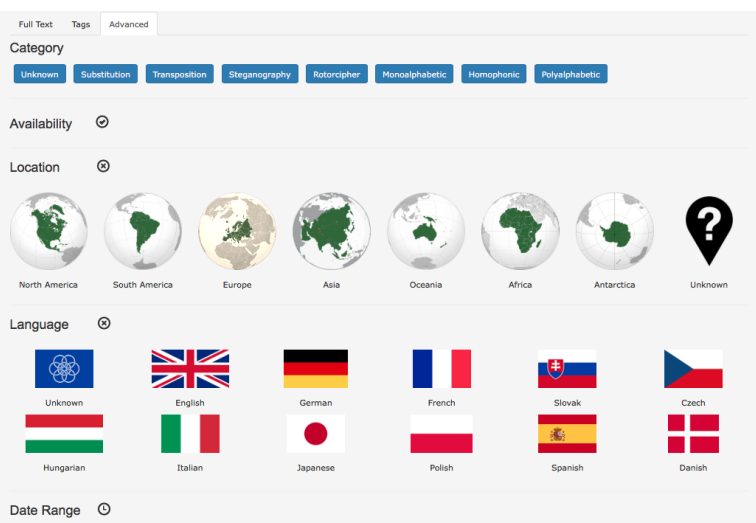

Figure 5: Cryptograms - advanced search options.

any operation realizable on a document transcription divided into a set of pages.

The implemented filters allow to change the reading direction, select sub-pages, or a subsection from the document, and calculate several statistics like the index of coincidence, Shannon's entropy, $n$-gram frequency, etc.

Later on, we have decided to create a more general framework independent from the operating systems, and ported the main functionality of the existing application to the web. ManuLab online is the online version of the ManuLab application, accessible via PHP scripts.

Furthermore we integrated the existing database of cryptograms directly to the example web page. The users can directly download and analyse text attachment of any cryptogram from the database.

The functionality was extended with cryptanalysis functions like language guess, anagram detection or Sukhotin's vowel detection method.

The source code is available online at the following GIT repository:

https://bitbucket.org/jugin/manulab. git.

The API documentation is available at: https://manulab.hcportal.eu/apidoc and an example web page (demonstrating the API) is available at:

https://manulab.hcportal. eu/example. 


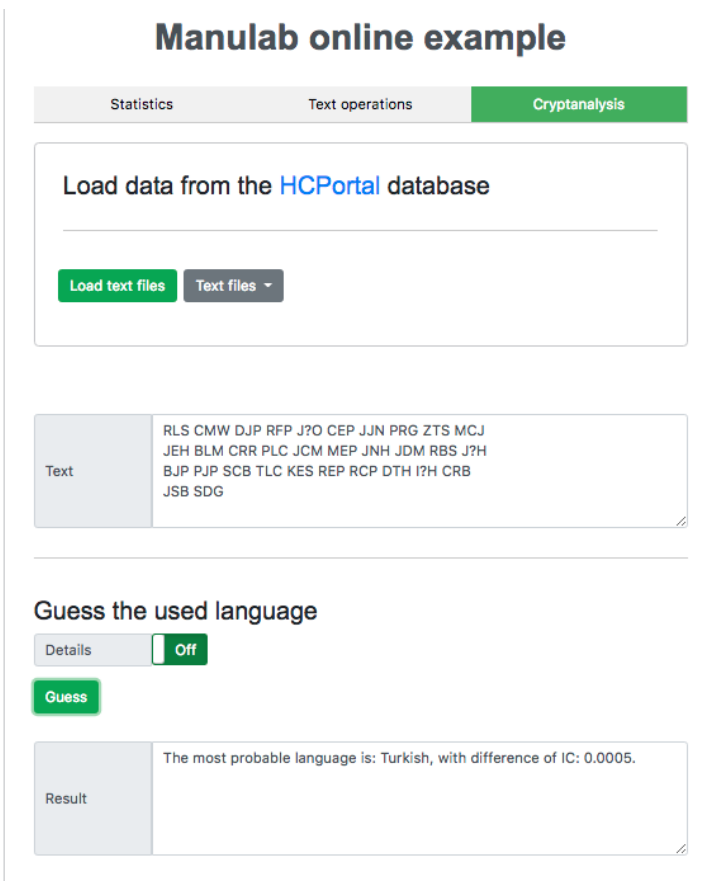

Figure 6: Manulab online API example.

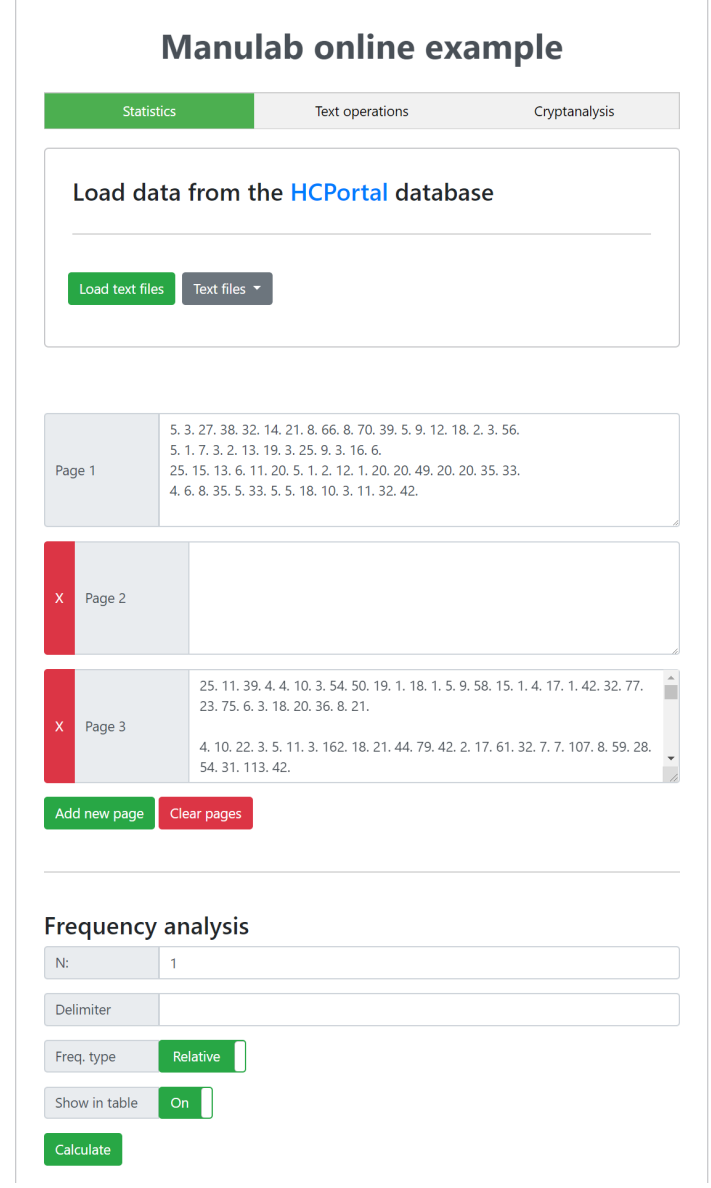

\section{Glossary}

This site contains definitions of terms related to historical cryptology, including terminology for codes and nomenclators. Terms related to modern cryptology are not covered. The used terms are mainly from the declassified Friedman's collection - Basic Cryptologic Glossary (REF ID:A64719) and from (Klausis Krypto Kolumne, 2019). We are currently collecting visual examples (pictures) of selected terms to extend this glossary.

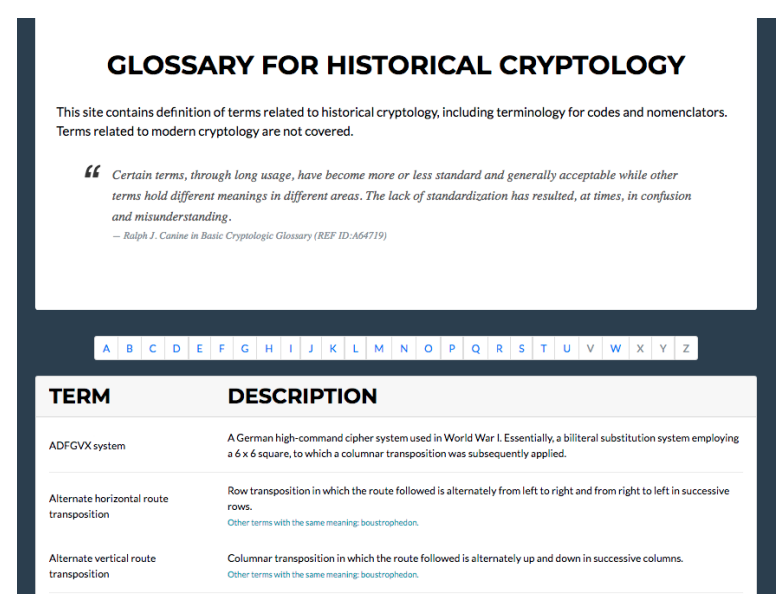

Figure 8: Glossary.

\section{Acknowledgments}

This work was partially supported by grants VEGA 1/0159/17 and VEGA 2/0072/20.

\section{References}

Klaus Schmeh. Klausis Krypto Kolumne http:// scienceblogs.de/klausis-krypto-kolumne

Frode Weierud. Crypto Cellar Research http:// cryptocellar.org/

Michael Hrenberg. Breaking German Navy Ciphers https://enigma.hoerenberg.com/

Satoshi Tomokiyo. Cryptiana http://cryptiana. web.fc2.com/code/crypto.htm

Figure 7: Manulab online API example - multiple input pages. 